LA- $-11627-M S$

DE90 000884

Glossary of Nuclear Criticality Terms

Hugh C. Paxton*

-Consultant at Los Alamos. 


\title{
GLOSSARY OF NUCLEAR CRITICALITY TERMS
}

by

Hugh C. Paxton

\begin{abstract}
This is a glossary of terms generally encountered in the literature of nuclear criticality and criticality safety. Terms sometimes misused are emphasized.
\end{abstract}

Potential value of a glossary of terms related to nuclear criticality is suggested by the observation that less than one-half of such terms in a 1988 manuscript on criticality accidents (D. R. Smith, "A Review of Criticality Accidents," DOE/NCT-04, 1989) are adequately defined in the 1986 Glossary of Terms in Nuclear Science and Technology. ${ }^{1}$ The present compilation may be considered an extension of that document to encourage the consistent vse of nuclear criticality terms.

The following pair of terms are so basic and so intertwined that they call for special consideration inconsistent with the body of this glossary. Consequently, they are given this introductory position.

critical, criticality: Proper use is generally consistent with the following definition from Webster's New International Dictionary, Second Edition, U.zabridged:

-ity. A suffix denoting state, condition, quality, or degree, used to form abstract nouns from adjectives, as in acidity, calamity. 
Thus, "delayed criticality" and "delayed critical state" are equivalent. "Critical" is not useu as a noun but may seem so by implying "critical state" in legends of graphs or charts where space is at a premium. When the meaning of "critical" as an adjective may be misinterpreted, as in "critical terms" or "critical accidents", "criticality" may be substituted for clarification. Use of "a criticality" for "a critical condition" or simply for "criticality," as is sometimes heard, is unacceptable. See delayed criticality. prompt criticality.

albedo, neutron: The probability under specified conditions that a neutron entering into a region through a surface will return through that surface.

absorbed dose: The energy imparted to matter by directly or ilisirectly ionizing radiation per unit mass of irradiated material at the point of interest; unit of absorbed dose has been the rad and now in the International System of Units (SI) is the gray (Gy), $100 \mathrm{rad}=1 \mathrm{~Gy}^{2,3}$ See rad. gray.

absorption, neutron: A neutron-induced reaction, including fission, in which the neutron disappears as a free particle. ${ }^{1}$ The absorption cross section is designated $\sigma_{\mathrm{a}}$. See capture, neutron; cross section. neutron.

alarm system, criticality accident: A system capable of sounding an audible alarm after detecting neutron or gamma radiation from a criticality accident. See criticality accident.

alpha particle: A helium-4 nucleus emitted during a nuclear transformation."

beta particle: An electron of either positive or negative charge that has been emitted in a nuclear transformation.

buckling: For our purposes, algebraic expressions that relate critical dimensions of various simple shapes (sphere, cylinder, or cuboid) of cores of the same composition and similar reflectors. For example, the known radius of a critical sphere may be used to obtain the radius and length of a corresponding critical cylinder. For a specific definition of buckling, see Ref. 4, pp 7 and 8 . See core, reflecior.

burst, prompt: Usually refers to the pulse of energy from fissions produced by a prompt burst reactor. See prompt burst reactor, spike (in $a$ prompt power excursion).

capture, neutron: Neutron absorption not leading to fission or other reutron production. The capture cross section is designated $\sigma_{c}$. See absorption, neutron; cross section. neutron.

cen:: A unit of reactivity equal to one-hundredth of the increment between delayed criticality and prompt criticality (a dollar).' See dollar. reactivity. 
chain reaction, fission: A sequence of nuclear fission reactions in which fissions are induced by neutrons emerging from preceding fissions. Depending on whether the number of fissions directly induced by neutrons from one fission is on the average less than, equal to, or greater than unity, the chain reaction is convergent (subcritical), self-sustaining (critical), or divergent (supercritical). ${ }^{1}$

core: That part of a fissile system containing most or all of the rissile material, as distinguished from an external reflector. See fissile system. reflector.

critical infinite cylinder. For specified fissile medium and surrounding reflector, the infinitely long cylinder with a diameter that would be critical.

critical infinite slab: For specified fissile medium and reflector on cach surface, the slab of infinite lateral dimensions with a thickness that would be critical.

criticality accident: The release of energy as a result of accidentally producing a self-sustaining or divergent fission chain reaction. ${ }^{1}$ See reaction, fission.

criticality safety: Protection from the consequences of a criticality accident, preferably by prevention of the accident. ${ }^{4}$ Encompasses procedures, training, and other precautions in addition to physical protection. See criticality accident.

criticality safety Standards: These Standards describe criticality control practices for which there is industry-wide consensus. Consensus is established through procedures of the American National Standards Institute. Chapter 4 of Ref. 4 lists and discusses existing and proposed criticality safety Standards, and explains capitalization of the term.

cross section $(\sigma)$, neutron: The proportionality factor that relates the rate of a specified reaction (such as capture or fission) to the product of the number of neutrons per second impinging normally onto a unit area of a thin target and the number of target nuclei per unit area. It may be considered a small area assigned to each target nucleus, usually expressed in barns, i.e., $10^{-24} \mathrm{~cm}^{2}$. See absorption. neutron; capture, neutron; fission. nuciear.

decay, radioactive: A spontaneous nuclear transformation in which particles or gamma radiation is emitted, in which x-radiation is cmitted following orbital electron capture, or in which the nucleus undergoes spontaneous fission." See fission, nuclear: gamma radiation.

delayed criticality: State of a fissile system such that k fff $^{=} 1$, the steady-state condition. See multiplication factor. 
delayed neutrons: Neutrons from nuclei produced by beta decay following fission. They follow fission by intervals of seconds to minutes. See prompt neutrons.

dollar: A unit of reactivity equal to the increment between delayed criticality and prompt criticality for a fixed chain-reacting system. See reactivity.

dose equivalent: The absorbed dose multiplied by the quality factor and other less significant modifying factors, so that doses from different radiations (alpha, beta, gamma, slow neutron, fast neutron) can be summed to provide an effective total dose at the point of interest. ${ }^{2}$ The conventional unit of dose equivalent has been the rem, and now in the International System of Units (SI) is the sicvert (Sv), $100 \mathrm{rem}=1 \mathrm{~Sv}^{5}$ See rem. sievert.

dose rate: Absorbed dose delivered per unit time. ${ }^{2}$ See absorbed dose.

excursion, nuclear: An episode during which the fission rate of a supercritical system increases, peaks, and then decreases to a low value.

excursion, prompt-power: A nuclear excursion as the result of a prompt-critical configuration of fissile material. In general, a sharp power spike followed by a plateau that may be interrupted by smaller spikes. See excursion, muclear: spike (in a frompt power excursion).

excursion period (T): The reciprocal coefficient of $t$, where fission power in a nuclear excursion increases as $\mathrm{e}^{\mathrm{t} / \mathrm{T}}$ before a quenching mechanism becomes effective. See excursion, nuclear; quenching mechanism.

exponential column: A subcritical block or cylinder of fissile-bearing material with an independent neutron source at one end. Under appropriate conditions, the response of a neutron detector decreases exponentially with distance from the source. From the logarithmic rate of this decrease and lateral dimensions of the column, critical dimensions of an unreflected assembly of the material may be deduced.

exposure: A measure of the ionization produced in air by $x$ rays or gamma radiation; the sum of electric charges on all ions of one sign in a small volume of air when all electrons liberated by photons are completely stopped, per unit mass of the air. Note that exposure refers to the environment, not absorbing material. The unit of exposure is the roentgen. ${ }^{2}$ See gamma radiation. roengen. Alternatively, exposure is the incidence of radiation on living or inanimate material."

fararable geometry: Geometric constraint of rissile matcrial in which subcriticality is maintained under anticipated conditions. Examples arc limited diameter of pipes intended to contain fissile solution, or limited volumes of solution containers. 
fissile nucleus: A nucleus rapable of fission by thermal neutrons, provided the effective neutron production cross section, $\overline{\nu \sigma}_{f}$, excecds the effective absorption cross section, $\bar{\sigma}_{a}$. The common fissile nuclei are ${ }^{235} \mathrm{U},{ }^{239} \mathrm{Pu}, \mathrm{nd}^{233} \mathrm{U}{ }^{3}$ Se absorption. neutron: fission, nuclear.

fissile system: A system containing ${ }^{235} \mathrm{U},{ }^{239} \mathrm{Pu}$, or ${ }^{233} \mathrm{U}$ nuclei and capable of significant ncutron multiplication. See fissile nucleus; multiplication, subcritical.

fission, nuclear: Disintegration of a nucleus (usually $T h, U, P u$, or heavier) into two (rarely more) masses of similar order of magnitude, accompanied by a large release of energy and the emission of neutrons. Although some fissions take place spontaneously, neutron-induced fissions are of major interest in criticality safety. The fission cross section is designated $\sigma_{f}$, and $\nu$ is the number of neutrons emitted per fission. See cross section. neutron.

fission products: Nuclides produced by fission ar by the subsequent radioactive decay of nuclides formed in this manner. ${ }^{1}$ See fission. nuclear: nuclide.

fission yield, excursion: The total number of fissions in a nuclear excursion. See excursion. nuclear.

fissionable nucleus: A nucleus capable of fission by neutrons of some energy. Fissionable nuclei include ${ }^{238} \mathrm{U},{ }^{240} \mathrm{Pu}$, and others with neutron-energy fission thresholds, in addition to those that are fissile. See fissile nucleus.

gamma radiation: Short-wavelength electromagnetic radiation emitted in the process of nuclear transition or particle annihilation.

gray (Gy): A unit of absorbed dose; $1 \mathrm{~Gy}=1 \mathrm{~J} / \mathrm{kg}=100$ rads. Adopted in 1976 by the International Conference on Weights and Measures to replace the rad. $^{5}$ See rad.

hazard: A potential danger. "Potentially hazardous" is redundant. Note that a hazardous facility is not necessarily a high-risk racility. See risk.

H/X: Conventionally, the atomic ratio of hydrogen to ${ }^{235} \mathrm{U}$, ${ }^{236} \mathrm{Pu}$, or ${ }^{233} \mathrm{U}$ in a solution or hydrogenous mixture. Where there is more than one fissile species, the ratios must be specified separately.

inhour: A unit of reactivity that when added to a delayed-critical system would produce a period of one hour; now seldom used.' See reactivity. 
ionizing radiation: Any radiation consisting of directly or indirectly ionizing particles, photons, or a mixture of both. $X$ rays and the radiations emitted in radioactive decay are examples." See decay, radioactive.

irradiation: Exposure to ionizing radiation.' See exposure (alternative definition).

isotopic code: Combined final digits of atomic number and atomic weight, such that ${ }^{235} \mathrm{U},{ }^{239} \mathrm{Pu}$, and ${ }^{233} \mathrm{U}$ are represented "25," "49," and "23"; ${ }^{240} \mathrm{Pu}$, however, is called "410"; these appear in some documents but now are seldom used.

linear energy transfer (LET): The average energy lost by an ionizing radiation per unit distance of its travel in a medium. A high LET is generally associated with protons, alpha particles, and neatrons, whereas a low LET is associated with $x$ rays, electrons, and gamma rays. ${ }^{2}$ See ionizing radiation.

monitor, radiation: A detector to measure the level of ionizing radiation. A purpose may be to give information about dose or dose rate. ${ }^{\prime}$ See ionizing radiation

multiplication, subcritical: In a subcritical fissile system containing a neutron source, the equilibrium ratio of the total number of neutrons resulting from fission and the source to the total number of neutrons from the source alone.'

multiplication factor ( $\left.k_{\text {eff }}\right)$ : For a chain-reacting system, the mean number of fission neutrons produced by a neutron during its life within the system. It follows that $k_{\text {eff }}=1$, if the system is criticai; $k_{\text {eff }}<1$, if the system is subcritical; $k_{\text {eff }}>1$, if the system is supercritical.

neutron: An elementary particle having no clectric charge, a rest mass of $1.67495 \times 10^{-24} \mathrm{~g}$, and a mean life of about $10 \mathrm{~min} .^{1}$

neutron poison: A nonfissionable ncutron absorber, generally used for criticality control. See absorption, neutron; capture. neutron.

neutrons, epithermal: Neutrons of kinetic cnergy greater than that of thermal agitation, often restricted to energies comparable with thuse of chemical bonds.'

neutrons, fast: Neutrons of kinetic energy greater than some specified value, of ten chosen to be $0.1 \mathrm{McV}$ (million elcctron volts).'

neutrons, thermal: Neutrons in thermal equilibrium with the medium in which they exist. ${ }^{9}$ At room temperature, the mean encrgy of thermal neutrons is about $0.025 \mathrm{eV}$ (electron volt). 
nonfarorable geometry: See favorable geometry.

nuclide: A species of atom characterized by its mass number, atomic number, and a possible clevated nuclear energy stat: if prolonged.'

oralloy (Oy): Introduced in carly Los Alamos documents to mean enriched uranium (Q $a k$ Ridge alloy); now uncommon except to signify highly enriched uranium. See tuballoy.

personnel monitor (radiation): A device for measuring a person's cxposure to radiation. Information on the dose equivalent of ionizing radiation to biological tissue is derived from exposures recorded by film badges, ionization chambers, and thermoluminescent devices; from whole-body counting and analysis of biological specimens; and from area monitoring and special surveys. ${ }^{2}$

photon: A quantum of electromagnetic radiation.'

prompt burst reactor: A device for producing nondestructive super-promptcritical nuclcar excursions. See burst, prompl; excursion, nuclear.

prompt criticality: State of a fissile system such that the prompt-neutron contribution to $k_{\text {eff }}$ equals unity. See multiplication factor.

prompt neutrons: Neutrons emitted immediatcly during the fission process. Ses delayed neutrons.

quality factor (QF): The linear energy-transfer-dependent factor by which absorbed doses are multiplied to obtain, for radiation-protection purposes, a quantity that expresses on a common scale the biological effectiveness of the absorbed dose derived from various radiation sources. ${ }^{2}$ Approximately the ratio of dose equivalent and absorbed dose. See absorbed dose. dose equivalent, linear energy transfer.

quenching mechanism: Physical process other than mechanical damage that limits an excursion spike. Examples are thermal expansion, or microbubble formation in a solution. See spike (in a prompt power excursion).

rad: A unit of absorbed dose; $1 \mathrm{rad}=10^{-2} \mathrm{~J} / \mathrm{kg}$ of the medium. In 1976, the International Conference on Weights and Measures adopted the gray $(1 G y=1 \mathrm{~J} / \mathrm{kg})$ as the preferred unit of absorbed dose, but this unit has not appeared in the criticality-accident literature, which was essentially complete before that date. See absorbed dose, gray, and discussion under personnel monitor.

radiation: In context of criticality safety, alpha particles, beta particles, neutrons, gamma rays, and combinations thereof. See alpha particle, bela particle. neutron, $x$ ray. 
reactivity: A parameter of a fissile system that is prcportional to $1-1 / k_{e f f}$. Thus, it is zero if the system is critical, positive if the system is supercritical, negative if the system is subcritical. See dollar, cent, and inhour, various units of reactivity; multiplication factor.

reflector: Matcrial outside the core of a fissile system capable of scattering back to the core some neutrons that would otherwise escape. See core, fissile system.

reflector savings: The absolute difference between a dimension of the reflected core of a critical system and the corresponding dimension of a similar core that would be critical if no reflector were present. ${ }^{1}$ See core, fissile system, reflector.

relative biological effectiveness (RBE): A factor used to compare the biological effectiveness of absorbed radiation doses (i.e., rads or grays) because of different types of ionizing radiation; more specifically, it is the experimentally determined ratio of an absorbed dose of a radiation in question to the absorbed dose of a reference radiation required to produce an identical biological effect in a particular experimental orgarism or tissue. ${ }^{3}$ This term should be used only in radiobiology, not instead of quality factor in radiation protection. See quality factor.

rem: A unit of dose equivalent (goentgen equivalent, man), replaced by the sievert, which was adopted in 1980 by the International Conference in Weights and Measures. ${ }^{5}$ This unit, however, has not appeared in the criticality-accident literature. See dose equivalent. sievert.

rep: An obsolete term for absorbed dose in human tissue, replaced by rad. Originally derived from roentgen equivalent, physical. ${ }^{1}$

risk: The cost of a class of accidents over a given period, usually expressed as dollars or fatalities, per year or during plant lifetime. Unless established by experience, risk is estimated as the product of the probability of occurrence and the consequence of the accident type. Not to be confused with hazard. See hazard.

roentgen ( $R$ ): A unit of exposure; $1 \mathrm{R}=2.58 \times 10^{-4} \mathrm{C} / \mathrm{kg}$ in air, where $C$ is coulombs. ${ }^{3}$ Strictly, the roentgen applies to $x$ rays or gamma radiation, although in one repor: of a criticality accident beta "dosages" are expressed in units of $R$. See exposure.

scram: An alternative term for reactor trip. ${ }^{1}$ Reference 6 gives accounts of the origin of this term.

shutdown mechanism: Quenching mechanism and mechianical damage, if any, that limits a prompt-power excursion spike. See excursion. prompt power: quenching mechanism; spike. 
sievert (Sv): A unit of dose cquivatent; $1 \mathrm{~Sv}=1 \mathrm{~J} / \mathrm{kg}=100 \mathrm{rcm}$. Adopted in 1980 by the International Conference on Weights and Measures to replace the rem. ${ }^{5}$ See dose equivalent, rem.

spike (in a prompt-power excursion): The initial power pulse of a prompt-power excursion, limited by the shutdown mechanism. See excursion. prompt power; shutdown mechanism.

tuballoy (Tu): A wartime term for natural uranium. originating in England; now obsoletc. See oralloy.

uranium enrichment (enrichment): The weight percentage of ${ }^{235} U$ in uranium, provided that percentage exceeds its natural value; if the reference is to enhanced ${ }^{233} \mathrm{U}$ content, "233 $\mathrm{U}$ enrichment" should be specified.

$x$ ray: Electromagnetic radiation of wavelength in the range $10^{-10} \mathrm{~cm}$ to $10^{-6} \mathrm{~cm} .^{7}$

\section{ACKNOWLEDGMENT}

The author wishcs to express his appreciation to Norman L. Pruvost and Michael J. Vehec for their assistance.

\section{REFERENCES}

1. Glossary of Terms in Nuclear Science and Technology. 1986 revision, prepared by ANS-9, the American Nuclear Society Standards Subcommittee on Nuclear Terminology and Units, Harry Alter, chairman, American Nuclear Society Publication, La Grange Park, IL.

2. "Terms Used in Radiation, Radiobiology, and Radioepidimiology," George L. Voelz, M.D., compiler, Group HSE-2, Los Alamos National Laboratory.

3. Radiological Health Handbook. Glossary pp 413-441, Revised Edition, January 1970, U.S. Department of Health, Education, and Welfare, Public Health Service, Consumer Protection and Environmental Health Service, Rockville, MD 20852.

4. Nuclear Criticality Safety Theory and Practice. Ronald A. Knief, American Nuclear Society, La Grange Park, IL (1985).

5. Dictionary of Scientific Units, H. G. Jerrard and D. B. MeNeill, Fifth Edition, Chapman and Hall, London (1972).

6. Raymond L. Murray, "The Etymology of 'Scram," Nuclear News, 3h, August 1988, pp 105-107; "Letters," Nuclear News. 31, December 1988, pp 17-18.

7. University Physics. 2nd Ed., F. W. Sears and M. W. Zemansky. Addison-Wesiey, Reading, MA (1955). 